\title{
El empleo de la historia de la Química como eje fundamental para su enseñanza en Educación Básica
}

\section{Diego Alejandro Barragán Currea ${ }^{4}$}

dqu383_dbarragan@pedagogica.edu.co

\section{Resumen}

El presente escrito pretende plasmar los hallazgos e intencionalidades que direccionaron la puesta en marcha de una propuesta investigativa, desarrollada en los espacios académicos de Práctica Pedagógica y Didáctica I y II. Se pretende, además, aportar algunos fundamentos en la construcción del concepto de Recurrencia Conceptual en Química y su implicación en el análisis de las perspectivas molares y moleculares de las sustancias en la enseñanza-aprendizaje de la Química.

\section{Palabras clave}

Recurrencia Conceptual, Concepciones en Química, Historia de la Química, Didáctica de la Química, Resolución de Problemas.

4. Estudiante del Departamento de Química - UPN 


\section{Justificación}

La presente investigación se fundamentó y se proyectó con el propósito de integrar las perspectivas históricas y epistemológicas a la enseñanza de las ciencias, así como los aportes que dichas perspectivas puedan contribuir en la construcción de modelos y metodologías didácticas propias para la enseñanza de las diversas disciplinas científicas, en general, y de la Química, en particular. Como ha sido considerado en las últimas reuniones, congresos y seminarios, entre otras actividades académicas, por parte de la comunidad de didactas de las ciencias, queda claro que la relación entre la historia y los diversos estatutos epistemológicos de las ciencias, y su correlación con la enseñanza, ocupan un lugar relevante, tanto en la investigación educativa contemporánea, como en la formación inicial de los futuros Licenciados.

En este sentido, el interés por indagar acerca de la recurrencia conceptual en la enseñanza de la Química resulta como un problema particular de la vinculación de dos núcleos investigativos primordiales. De una parte, las problemáticas alrededor de las ideas alternativas que han sido mostradas y caracterizadas por la investigación didáctica contemporánea, a la vez que ha sido indicada la influencia que tienen estas ideas o concepciones en los procesos de enseñanza-aprendizaje. De otra parte, los análisis históricos y epistemológicos, que a propósito de las ciencias han sido desarrollados durante el siglo $\mathrm{XX}$, han mostrado las características epistémicas y experimentales particulares de cada una de las disciplinas del conocimiento científico, señalándose, igualmente, nuevos problemas didácticos al integrarse dichas reflexiones al campo de la enseñanza de las ciencias. (Martínez,. 2005).

En este sentido, se presentan los presupuestos conceptuales y teóricos en el campo de la epistemología y la historia de las ciencias, así como aquellos derivados de la discusión que se ha suscitado en el campo de la didáctica de las ciencias, a propósito de las particularidades del conocimiento químico; su peculiar estatuto epistemológico, que indica supervivencias conceptuales que no suponen reemplazos teóricos totales; la caracterización de los modos de representación y modelización desde la historia de la Química, así como los núcleos de debate acerca de la posibilidad de que el manejo conceptual y metodológico, en Química, dependa de la resolución de problemas en que se tenga interés.

El problema de las concepciones en Química enfatiza en el campo de la estructura de las sustancias y de los modelos moleculares, en donde se pueden categorizar modos de comprensión de la estructuración química de las sustancias, y se puede hacer más palpable la problemática de la recurrencia conceptual.

\section{Posibilidad de coexistencia de concepciones diferentes sobre un mismo tópico}

Los estudios sistemáticos llevados a cabo sobre la influencia del contexto en el uso de concepciones a través de distintas tareas, constituyen un buen ejemplo que ilustra uno de los tipos de datos empíricos que han servido de apoyo para sustentar la hipótesis constructivista de las concepciones espontáneas. En efecto, algunos autores y autoras (Clough y Dríver, 1986; Jiménez-Aleixandre y Fernández, 1989; Oliva, 1994) han utilizado los criterios de consistencia como argumento para defender la existencia de concepciones firmemente asumidas por los alumnos, frente a la hipótesis trivial que ha visto en ellas simples contestaciones ad hoc, generadas para salir al paso de las cuestiones formuladas.

A pesar de conseguir sus objetivos parcialmente, los trabajos de este tipo han mostrado una realidad más compleja de la que en principio se esperaba, con niveles estadísticamente significativos de consistencia en los esquemas utilizados, y, 
al mismo tiempo, con variaciones importantes en las respuestas emitidas. Si bien las ideas de los alumnos no son producto de respues 7 tas aleatorias, su existencia tampoco se explica satisfactoriamente desde una visión constructivista, centrada en la hegemonía de esquemas de conocimiento únicos perfectamente establecidos y asentados. Ello ha contribuido a abrir un debate en torno al diferente grado de compromiso cognitivo que el alumno mantiene con sus concepciones en distintos casos. En unas ocasiones, esto podría ser el reflejo de un conocimiento interno verdaderamente estable, mientras que en otras podría estar condicionado por una forma excesivamente directiva de plantear las tareas que sirven para su detección.

Autores como Marión (1981) ya mencionaban, hace unos años, la existencia de variaciones en las concepciones para un mismo fenómeno, no solo entre niños distintos, sino incluso a nivel individual en función del contexto involucrado. Según esta hipótesis, que ha sido contrastada con posterioridad en los trabajos sobre coherencia y consistencia en las ideas, las concepciones no serían consecuencia de los rasgos de un individuo, sino que son características de las rotaciones entre el individuo, el contenido y el contexto. Línder (1993) denomina a este fenómeno "dispersión conceptual” y muchos autores se refieren a él cuando sugieren la posibilidad de que puedan coexistir en un mismo alumno distintas concepciones que compiten ante una situación determinada (Maloney y Siegler, 1993).

Al hablar de coexistencia de concepciones no solo se hace referencia a que, después de la instrucción, los alumnos compartan una visión científica y una visión intuitiva previa a la enseñanza, sino también a que ya, desde una fase anterior al aprendizaje escolar, un mismo alumno pudiera compartir diversos esquemas alternativos sobre un mismo tópico que rivalizan y compiten entre sí.

\section{Sobre la recurrencia conceptual}

El problema de la recurrencia se extiende a la discusión acerca de las concepciones en Química y su relación con la resolución de problemas de la disciplina. Como lo han mostrado los análisis históricos y epistemológicos, subsisten en el pensamiento químico diversos modos de representación y modelización (Greca, I.M. \& Dos santos, F., 2005), que ofrecen posibilidades teóricas y metodológicas para dar una explicación satisfactoria, y cuya diferencia fundamental radica en su estatuto históricoepistemológico. Por recurrencia conceptual podría entenderse, entonces, la elección que los sujetos realizan de un conocimiento cuando han de enfrentarse a una problemática, así como la conveniencia que ellos encuentran en los esquemas conceptuales y metodológicos, sobre otros, igualmente satisfactorios, para la cuestión planteada.

Desde esta perspectiva, el problema que propone la construcción del concepto de recurrencia conceptual, se pone de manifiesto al considerar las características epistemológicas propias y diferenciales de los conocimientos disciplinarios, que proveen diversas explicaciones completas y satisfactorias a hechos y fenómenos, revelándose que en la resolución de un problema puede recurrirse a conceptos y teorías basadas en distintas concepciones.

En el caso de la Química este problema resulta importante, pues la discusión epistemológica contemporánea ha mostrado la subsistencia de concepciones que se creen históricamente superadas, pero que influyen en los procesos de representación y modelización de los químicos. Con base en estos presupuestos históricos y epistemológicos, el problema didáctico emerge al considerarse que, en la resolución de ciertos problemas en Química, los estudiantes pueden recurrir a explicaciones de diverso tipo, con fundamentos teóricos y experimentales satisfactorios frente a la cuestión plantea- 
da, no pudiéndose establecer exclusión total entre ellos aunque puedan derivar de modelos diferentes (Martínez, 2005).

\section{Concepciones en Química: perspectivas históricas y didácticas}

La diferenciación y caracterización de las diversas concepciones que han hecho parte del proceso histórico de construcción de conocimiento en Química, así como las cuestiones de enseñanza derivadas de estos análisis, poseen una amplia extensión en la literatura disponible (Gabel, 1998; Johnstone, 2000). Nelson (2002) señala que la Química obedece a tres niveles de descripción: macroscópico, atómico-molecular y electrónico-nuclear. Para el autor, la lógica que establece este orden, no solo establece una secuenciación cronológica del desarrollo de los principales aportes teóricos en la historia de la Química (establecimiento de elementos químicos en el siglo XVIII, la teoría atómica en el siglo XIX y las teorías electrónicas en el siglo $X X)$, sino que, además, indica una lógica adoptada para la organización curricular de la Química, que fue adoptada desde principios de la década de los sesenta, y cuyo abandono ha resultado en los numerosos ejemplos de errores conceptuales que provee la literatura en didáctica de la Química (Nelson, 2002:216). Desde su perspectiva, retomar los procesos de construcción de conocimiento, siguiendo el orden antes mencionado, se constituye en una alternativa para la enseñanza de la Química, con base en los desarrollos del conocimiento moderno.

Jensen (1998) propone tres aproximaciones conceptuales según las cuales han sido construidos los modelos y conceptos en Química. La aproximación molar, que trata con las propiedades químicas macroscópicas de las sustancias; la aproximación molecular, que trata con las propiedades químicas de la estructura e interacción entre las moléculas y la aproximación eléctrica, que trata con las propiedades derivadas de las interacciones electrónicas y nucleares en los átomos y moléculas. De igual manera, Jensen señala que la aparición de estas aproximaciones conceptuales se corresponde con una revolución científica en la historia de la Química. Para Wu, Krajcik y Soloway (2000), estas aproximaciones conceptuales corresponden con las representaciones macroscópicas, microscópicas y simbólicas, propuestas analizadas que constituyen interpretaciones de los químicos acerca de la naturaleza y su uso refleja las teorías, los hallazgos y los descubrimientos en Química.

\section{Problema}

En la presente investigación, el problema se extiende a la discusión acerca de las concepciones en Química y su relación con la resolución de problemas en esta disciplina.

Como lo han mostrado los análisis históricos y epistemológicos, subsisten en el pensamiento químico diversos modos de representación y modelización, que ofrecen posibilidades teóricas y metodológicas para dar una explicación satisfactoria; la diferencia fundamental entre ellos radica en su estatuto histórico-epistemológico.

El problema de investigación didáctica estriba, de esta manera, en la indagación de los problemas frente a los cuales pueden existir esquemas teórico-conceptuales y metodológicos venidos de formas diferentes de representación y modelización científica en Química, estableciendo tendencias de recurrencia a unas u otras, así como señalando las implicancias de orden didáctico y las posibilidades de intervención y diseño curricular.

En la presente investigación, el problema se extiende a la discusión acerca de las concepciones en Química y su relación con la resolución de problemas en esta disciplina.

Como lo han mostrado los análisis históricos y epistemológicos, subsisten en el pensamiento químico diversos modos de representación y modelización, que ofrecen posibilidades 
teóricas y metodológicas para dar una explicación satisfactoria; la diferencia fundamental entre ellos radica en su estatuto históricoepistemológico.

El problema de investigación didáctica estriba, de esta manera, en la indagación de los problemas frente a los cuales pueden existir esquemas teórico-conceptuales y metodológicos venidos de formas diferentes de representación y modelización científica en Química, estableciendo tendencias de recurrencia a unas u otras, así como señalando las implicancias de orden didáctico y las posibilidades de intervención y diseño curricular.

De igual manera, se busca dar una explicación de los factores que influyen en la elección del conocimiento que hacen los sujetos, la concordancia con sus intereses y conveniencias, y las posibilidades que en este orden facilitan procesos de cambio conceptual. La investigación reciente en didáctica de la Química ha dado cuenta, no solo de la coexistencia de diversas concepciones para responder por problemáticas propias de la Química, sino que la utilización de unas u otras no puede explicarse simplemente por el reemplazo de las históricamente mejor elaboradas frente a las anteriores.

En este sentido, los problemas que subyacen a la investigación, consisten en determinar, en primer lugar, si es posible la construcción de un concepto como el de recurrencia conceptual, que vincule las características histórico-epistemológicas del conocimiento químico, con las concepciones que poseen los sujetos cuando se enfrentan a la resolución de problemas en la enseñanza-aprendizaje de la Química a nivel de la educación media. En segundo lugar, examinar si logra evidenciarse y evaluarse la recurrencia a conceptos y teorías derivados de diversas concepciones, para enfrentar un problema en Química por parte de una población escolar de educación media; finalmente, se busca determinar si es posible pensar en la existencia de una recurrencia a nivel metodológico en el aula de clases.

\section{Conclusiones}

Al interrelacionar la historia de una disciplina con su enseñanza queda palpablemente evidenciado que es una alternativa para generar un aprendizaje significativo, ya que se puede apelar a la enseñanza del estatuto epistémico que posea mayor construcción, aportando herramientas utilizables para la resolución de problemas y una mejor comprensión de una disciplina científica con las peculiaridades de coexistencia de concepciones como lo presenta la Química.

La construcción del concepto de Recurrencia Conceptual está muy lejos de llegar a su fin y puede ser empleado para la construcción de metodologías didácticas diferenciales para la enseñanza particular de la Química.

Es posible complementar las relaciones históricas y epistemológicas con el desarrollo profesional de un docente en formación inicial, ya que este pone en juego sus propias concepciones y su construcción teórica, que ha logrado construir enfrentándose al discurso validado por la comunidad de especialistas y sus contribuciones a la investigación contemporánea del aula.

\section{Bibliografía}

Grupo de investigación Pensamiento, Ciencia y Enseñanza (2004). Proyecto de Investigación DQU-040-04. La Enseñanza Teórica de la Estructura de las sustancias, Informe Final, pp.15-24. Bogotá: Universidad Pedagógica.

Jensen, W.B. (1998). Logic, history and the chemistry textbook. Journal of Chemical Education, 75, 679-687,817-828,961-969.

Oliva, J. (1999). Algunas reflexiones sobre las concepciones alternativas y el cambio conceptual. Enseñanza de las Ciencias, 17(1), 93-107. 